\title{
Pulmonary Nodular Amyloidosis in Sjögren Syndrome
}

ROBERTO CARBONE, MD, Respiratory Unit, Department of Internal Medicine, Regional Hospital, Aosta; CLAUDIO COSSO, MD; MARCO A. CIMMINO, MD, Associate Professor, Research Laboratory and Academic Unit of Clinical Rheumatology, Department of Internal Medicine, University of Genoa, Genoa, Italy. Address correspondence to Dr. M.A. Cimmino, Viale Benedetto XV, 6 - 16143, Genoa, Italy. E-mail: claudio.cosso86@gmail.com.

J Rheumatol 2015;42:134; doi:10.3899/jrheum.140964

Pulmonary involvement because of amyloidosis is rare in Sjögren syndrome (SS). It can be treated with corticosteroids.

A 40-year-old woman presenting with dry cough, dyspnea score Moser 1, chest pain, and fatigue was investigated for suspected interstitial pulmonary disease. Fifteen years earlier, she had been diagnosed with SS on the basis of sicca syndrome, positive salivary glands biopsy, antinuclear antibody $>$ 1:640 (granular pattern), and high titer anti-La and anti-Ro antibodies. Physiological lung function showed an initial restrictive pattern with normal carbon monoxide transfer. High-resolution computed tomography (HRCT) identified multiple pulmonary nodules (Figure 1A), which showed intense uptake on a positron emission tomography/CT scan (Figure 1B). QuantiFERON (Quest Diagnostics) test was negative. To exclude possible lung cancer, a biopsy of one of the nodules was performed. Histopathological evaluation showed peribronchial and perivascular lymphoid nodular hyperplasia, with perivascular and interstitial amyloid deposits seen after use of dye (Figure 1C, arrows). The patient was prescribed prednisone at an initial dose of $25 \mathrm{mg}$ daily for 1 month, which was tapered over 6 months to $5 \mathrm{mg}$ daily with a complete resolution of the symptoms.

Pulmonary involvement because of amyloidosis is rare in SS, occurring in only $2 / 343$ of the patients $(0.6 \%)$ studied by Strimlan, et $\mathrm{al}^{1}$. A biopsy of nodular amyloidosis of the lung has revealed amyloid light-chain type ${ }^{2}$ or transthyretin amyloidosis $^{3}$.

\section{REFERENCES}

1. Strimlan CV, Rosenow EC 3rd, Divertie MB, Harrison EG Jr. Pulmonary manifestations of Sjögren's syndrome. Chest 1976;70:354-61.

2. Miyagawa T, Mochizuki Y, Nakahara Y, Kawamura T, Sasaki S, Kobashi Y. [A case of Sjögren syndrome with pulmonary nodular amyloidosis and pulmonary multiple cysts]. [Article in Japanese] Nihon Kokyuki Gakkai Zasshi 2009;47:737-41.

3. Sakai T, Tsushima T, Kimura D, Fukuda I, Kamata Y, Hatanaka R, et al. [Multiple nodular pulmonary amyloidosis complicated with Sjögren syndrome]. [Article in Japanese] Kyobu Geka 2010;63:818-21.

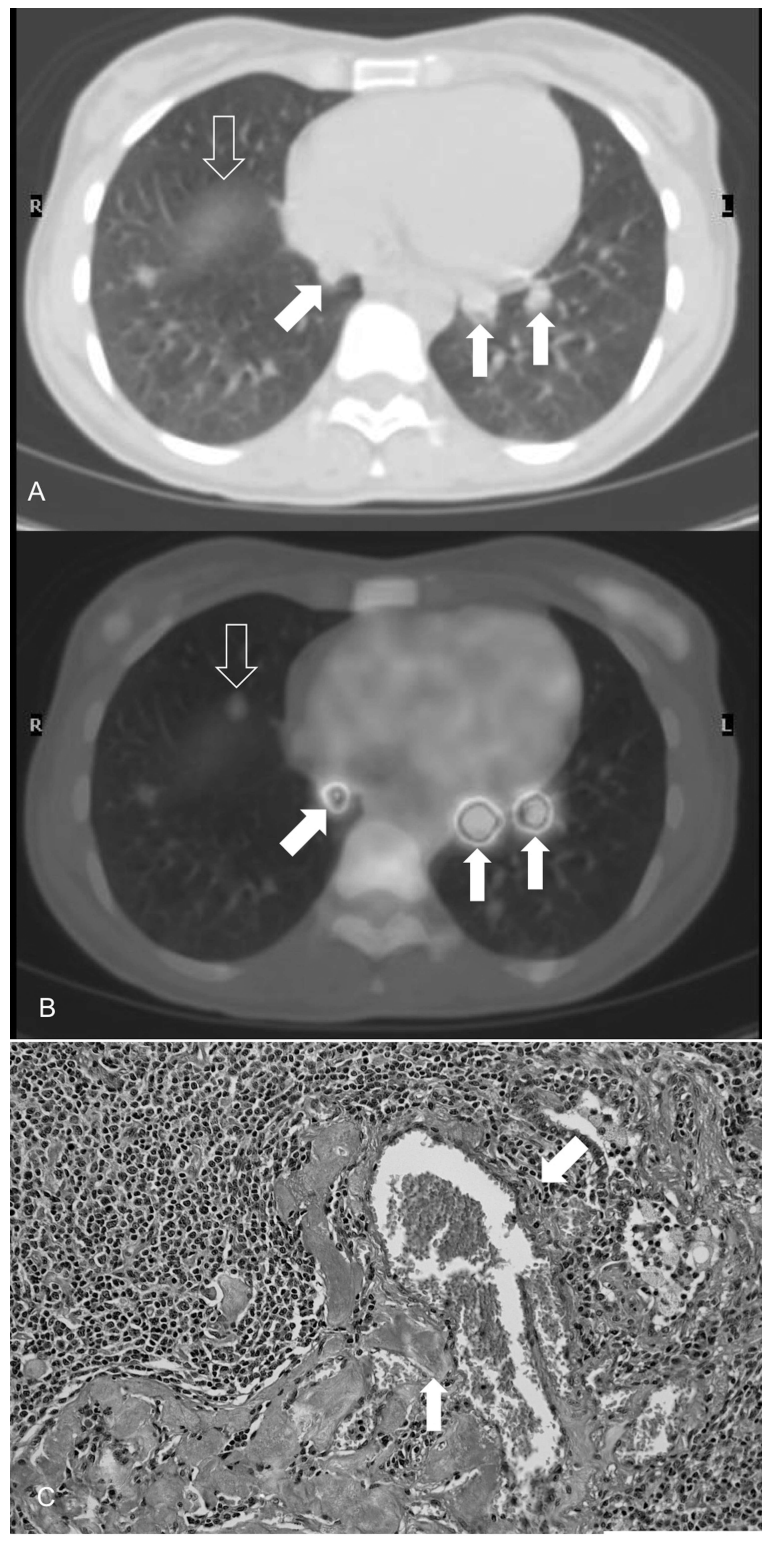

Figure 1. A. High-resolution computed tomography identified multiple pulmonary nodules. B. Nodules showed intense uptake on a positron emission tomography/computed tomography scan. C. Histopathological evaluation showed peribronchial and perivascular lymphoid nodular hyperplasia, with perivascular and interstitial amyloid deposits seen after use of dye (arrows). 